\title{
A Particle Extraction Method with an Improved Auto-encoder in Neural Networks with the Aid of HOG Feature Analysis
}

\author{
Shoki Tezuka ${ }^{1}$, Gen Maeda ${ }^{1}$, Misuzu Baba ${ }^{2}$ and Norio Baba ${ }^{1}$ \\ 1. Major of Informatics, Graduate School, Kogakuin University, Shinjuku, Japan \\ 2. Research Institute for Science and Technology, Kogakuin University, Hachioji, Japan
}

In the field of electron microscopic image analysis, machine learning such as neural networks [1] is remarkable method, which has abilities of automatic extraction of structural objects and automatic classification. Recently, deep leaning in the neural networks is actively applied because of high recognition rate [e.g. 2]. This learning method is able to choose effective feature patterns from a set of learned local images. We are developing a deep learning software for application to biological transmission electron microscope images (including electron tomographic images), especially yeast cell (Saccharomyces cerevisiae) images, in which automatic particle extraction is a purpose. We already applied a preliminary software to ultra-thin section images of the yeast cell for picking out some kinds of structural particles (e.g. Virus-like particle (VLP) in autophagy). The characteristics of the software is that it is made of auto-encoder in neural networks. The image analysis with the auto-encoder is essentially equivalent to that with the principal component analysis (PCA) [3]. The auto-encoder analyses a lot of particle images which are manually collected and a certain number of important image components are automatically extracted from the particle images like PCA. These components are visualized and the number of components is adjustable. We are improving the function of the autoencoder so as to raise the efficiency.

We are also developing a particle collection assist software by using the HOG (Histograms of Oriented Gradients) feature analysis which makes the manual supervised learning easy. The HOG feature analysis is based on the idea that local object appearance and shape can often be characterized rather well by the distribution of local intensity gradients or edge directions [4]. At present, the analysis is well used for human detection in pictures of a monitoring camera. The biological particles such as VLP have a unique shape (like a circular ring). Therefore, this feature seemed to be suitable for the analysis. In practice the analysis is implemented by dividing the image window into small spatial regions ("cells"), for each cell accumulating a local 1-D histogram of gradient directions or edge orientations over the pixels of the cell. In the general picture analysis field, for better invariance to illumination, shadowing, etc., it is also useful to contrast-normalize the local responses before using the direct results. However, in the electron microscopic image experiments, the normalization was not needed, which rather suppressed the feature differences. In the practical application of the HOG, various sizes of the cell were tested. As a result, a small size less than the particle was effective. The HOG-based automatic VLP extraction was examined. A preliminary result is shown in the figures.

Fig.1 shows the examined electron microscope image of yeast cell. Many virus-like particles (VLP) in autophagy can be observed, which are buried in the ribosome rich cytoplasm. Fig.2 shows a result of the HOG-based VLP extraction. The automatic extraction was not completed, but it usefully assisted the supervised leaning for the auto-encoder pre-learning. Fig.3 shows an example of the final result with the auto-encoder, which will be improved further in proportion to the pre-leaning with the aid of the HOGbased auto extraction. 


\section{References:}

[1] Ogura T et al, Journal of Structural Biology 136 (2001), p. 227.

[2] Chklovskii D B et al, Neurobiology 20 (2010), p. 1.

[3] Baldi P and Hornic K, Neural Networks 2 (1989), p. 53.

[4] Dalal N and Triggs B, IEEE Computer Society Conference on CVPR, (2005)

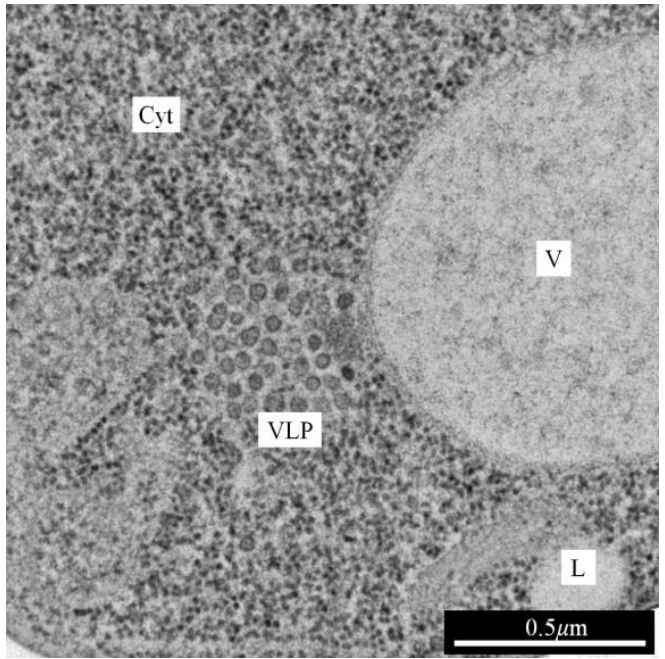

Figure 1. Examined electron microscope image of yeast cell. Many virus-like particles (VLP) in autophagy can be observed, which are buried in the ribosome rich cytoplasm.

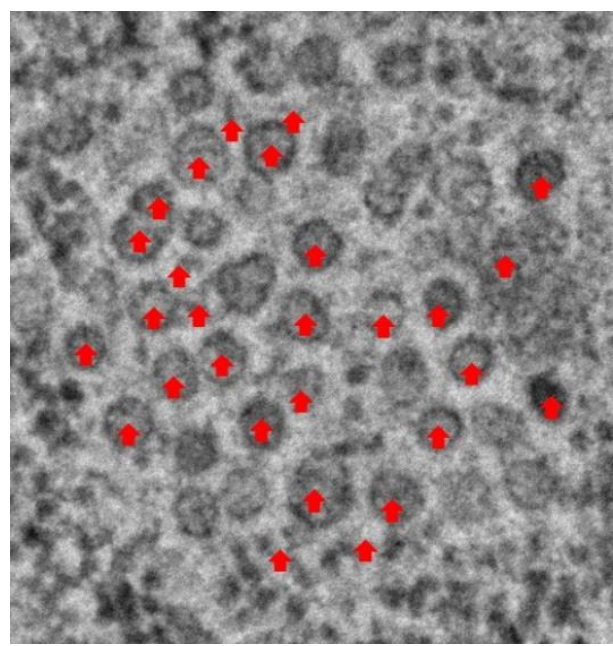

Figure 2. A result of the HOG-based VLP extraction (red marks).

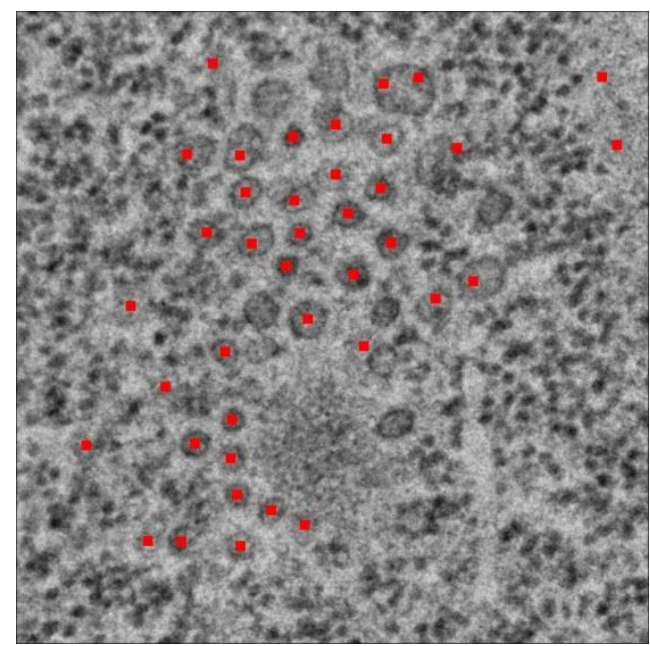

Figure 3. An example of the final VLP extraction result with the auto-encoder. 